\title{
MicroRNA-212-3p inhibits paclitaxel resistance through regulating epithelial-mesenchymal transition, migration and invasion by targeting ZEB2 in human hepatocellular carcinoma
}

\author{
JIANYU YANG ${ }^{1,2^{*}}$, RONGHUA CUI ${ }^{3 *}$ and YINGKE LIU $^{4}$
}

\begin{abstract}
${ }^{1}$ Workshop of National TCM Master, Sun Guangrong, The Harmonizing School of TCM, Beijing University of Chinese Medicine; ${ }^{2}$ Inheritance Workshop in Beijing Hepingli Hospital for National TCM Master, Sun Guangrong; ${ }^{3}$ Department of Oncology, Affiliated Haikou Hospital of Xiangya Medical College, Central South University, Haikou, Hainan 570208; ${ }^{4}$ Department of Pediatrics, Beijing Hepingli Hospital, Beijing 100013, P.R. China
\end{abstract}

Received January 11, 2019; Accepted April 1, 2020

DOI: $10.3892 / \mathrm{ol} .2020 .11884$

\begin{abstract}
Hepatocellular carcinoma (HCC) is one of the most common tumor malignances with poor chemotherapeutic efficiency due to chemoresistance. MicroRNAs (miRNAs) have essential roles in regulating chemoresistance. However, the mechanism underlying the involvement of miR-212-3p in paclitaxel (PTX) resistance in HCC remains unclear. PTX resistance was investigated in the present study by assessing cell viability, the half maximal inhibitory concentration of PTX, resistance-associated protein levels and apoptosis. The expression levels of miR-212-3p and zinc finger E-box binding homeobox 2 (ZEB2) were detected by reverse transcription-quantitative PCR and western blotting. The epithelial-mesenchymal transition (EMT), migration and invasion were evaluated by western blotting and transwell assay. The association between miR-212-3p and ZEB2 was investigating by the luciferase activity. The results showed that treatment of HCC cells with PTX inhibited cell viability and miR-212-3p level. Moreover, miR-212-3p was reduced and its overexpression resulted in decreased cell viability, half maximal inhibitory concentration $\left(\mathrm{IC}_{50}\right)$ of PTX and levels of P-glycoprotein and glutathione S-transferase $\pi$, but increased cell apoptosis, in Huh7/PTX cells. However, miR-212-3p knockdown induced opposite effects in Huh7 cells. Furthermore, EMT, migration and invasion were induced in Huh7/PTX cells and the addition of miR-212-3p inhibited EMT, migration and invasion. Meanwhile, miR-212-3p abrogation caused the opposite effects in Huh7 cells. Additionally,
\end{abstract}

Correspondence to: Dr Yingke Liu, Department of Pediatrics, Beijing Hepingli Hospital, 18 North Hepingli Street, Dongcheng, Beijing 100013, P.R. China

E-mail: etwew321@126.com

${ }^{*}$ Contributed equally

Key words: ZEB2, microRNA-212-3p, hepatocellular carcinoma, paclitaxel
ZEB2 was directly targeted by miR-212-3p and its restoration or silencing abated the effect of miR-221-3p overexpression or knockdown in Huh7/PTX or Huh7 cells, respectively. The data from the present study suggest that miR-212-3p attenuates PTX resistance, by regulating EMT, migration and invasion via targeting ZEB2 in HCC cells, indicating a novel target for HCC chemotherapy.

\section{Introduction}

Hepatocellular carcinoma (HCC) has developed into one of the most important medical problems, with high incidence (6th in terms of new cases) and mortality rates (5-year survival rate is $\sim 18 \%$ ) worldwide in recent years (1). With advances in understanding the pathogenesis of HCC, many treatments have been used to increase survival in patients with HCC, including surgical treatment, radiotherapy and chemotherapy (2). Emerging evidence suggests that many chemotherapeutic drugs, including cisplatin, sorafenib and paclitaxel (PTX), are used for the treatment of HCC, however, resistance is regarded as a major hindrance of these drugs in $\mathrm{HCC}(3,4)$. Hence, much hope is placed in probing novel target to ameliorate resistance to PTX in HCC.

MicroRNAs (miRNAs/miRs) are a class of small non-coding RNAs with 20-25 nucleotides, which play essential roles in the diagnosis and prognosis of HCC via multiple pathways (5). Moreover, miRNAs have vital roles in PTX resistance in HCC by regulating different molecular signaling pathways (6). For instance, miR-877 was found to regulate PTX sensitivity in HCC by targeting forkhead box protein M1 (FOXM1) (7). In addition, miR-153 contributes to resistance of HCC cells to chemotherapeutic agents, such as sorafenib, etoposide and PTX (8). As for miR-212-3p, a miRNA plays an important role in cancer progression by regulating cell proliferation and apoptosis (9). Furthermore, previous study suggested that miR-212 is associated with radio-sensitivity in glioma cells by regulating breast cancer susceptibility gene 1 (BRCA1) (10). Besides, miR-212 could suppress cell proliferation and promote cell apoptosis by regulating FOXA1 in HCC (11). Notably, miR-212-3p, a mature form of miR-212, is expressed and may be used as a potential target for the diag- 
nosis, prognosis and treatment of HCC (12). However, there is no direct evidence that miR-212-3p participates in resistance to PTX in HCC.

Zinc finger E-box binding homeobox 2 (ZEB2) has been reported as a transcription factor, which exerts an important impact on the development of the nervous system (13). Moreover, ZEB2 is a key factor of epithelial-mesenchymal transition (EMT), which is associated with resistance to cisplatin or PTX in human lung cancer cells (14). Notably, ZEB2 plays essential roles in HCC progression, through regulating EMT, invasion and metastasis (15). Intriguingly, bioinformatics analysis using TargetScan provided putative binding sites of miR-212-3p and ZEB2. Hence, it was hypothesized that ZEB2 may be involved in miR-212-3p-mediated PTX resistance in HCC. In the present study the expression of miR-212-3p, EMT, migration and invasion were assessed in PTX-resistant HCC cells. Moreover, the effect of miR-212-3p on PTX resistance, EMT, migration and invasion were investigated. In addition, the association between miR-212-3p and ZEB2, and their effect on PTX resistance, was explored in HCC cells.

\section{Materials and methods}

Cell culture and treatment. The human HCC cell lines Huh7 and HCCLM3 cells were purchased from Japanese Collection of Research (JCRB Cell Bank, Japan). All cells were maintained in RPMI-1640 culture medium (Gibco; Thermo Fisher Scientific, Inc.) containing $10 \%$ fetal bovine serum (Gibco; Thermo Fisher Scientific, Inc.), $1 \%$ penicillin and streptomycin (Invitrogen; Thermo Fisher Scientific, Inc.) at $37^{\circ} \mathrm{C}$ with $5 \% \mathrm{CO}_{2}$ during the study. The PTX-resistant Huh7 cells were constructed by continuous treatment for $24 \mathrm{~h}$ at $37^{\circ} \mathrm{C}$ with increasing concentrations of PTX $(0-20 \mathrm{nM})$ and removing any dead PTX-sensitive cells. Briefly, Huh7 cells were treated with $1 \mathrm{nM}$ PTX for $24 \mathrm{~h}$. Subsequently, the medium was replaced with fresh PTX-free medium. After cells reached $80 \%$ confluence, they were incubated with gradually increasing concentrations of PTX $(2,5,10$ and $20 \mathrm{nM})$ for $24 \mathrm{~h}$ at $37^{\circ} \mathrm{C}$, and PTX-resistant Huh7 cells were collected (resistant to 20 nM PTX). Subsequently, PTX-resistant cells (Huh7/PTX) were cultured in RPMI-1640 medium with 10 nM PTX.

Cell transfection. miR-212-3p mimic (miR-212-3p; 5'-UA ACAGUCUCCAGUCACGGCC-3'), mimic negative control (miR-NC; the non-targeting sequence, 5'-CGAUCGCAUCA GCAUCGAUUGC-3'), miR-212-3p inhibitor (5'-GGCCG UGACUGGAGACUGUUA-3'), inhibitor negative control (miR-NC inhibitor; the non-targeting sequence, 5'-CUAAC GCAUGCACAGUCGUACG-3'), pcDNA3.1-ZEB2 overexpression vector (ZEB2), pcDNA3.1 vector (Thermo Fisher Scientific, Inc.) alone as overexpression vector negative control (vector), small interfering (si)RNA against ZEB2 (siZEB2; 5'-UGAUAUUGUUUCUCAUUCGGC-3'), and siRNA negative control (scrambled; 5'-UUCUCCGAACGUGUCACGU TT-3') were obtained from Shanghai GenePharma Co., Ltd. Transient transfection of Huh7 and Huh7/PTX cells with $40 \mathrm{nM}$ oligonucleotides or vector was performed by using Lipofectamine $^{\mathrm{TM}} 2000$ (Invitrogen; Thermo Fisher Scientific, Inc.), according to the manufacturer's instructions. Cells were collected for further analyses after $48 \mathrm{~h}$ post-transfection.
Cell viability assay. Cell viability was measured by using the 3-(4, 5-dimethylthiazol-2-yl)-2, 5-diphenyl-trtrazolium bromide (MTT) assay. Briefly, Huh7 and Huh7/PTX cells were seeded into 96-well plates at the density of $1 \times 10^{4}$ cells/well and incubated with varying concentrations of PTX for $24 \mathrm{~h}$. Each sample was conducted in triplicate. Then cell medium was replaced with $0.5 \%$ MTT (Sigma-Aldrich; Merck KGaA) and incubated for another $4 \mathrm{~h}$ at $37^{\circ} \mathrm{C}$. After the removal of the supernatant, dimethyl sulfoxide (100 $\mu \mathrm{l} /$ well; Sigma-Aldrich; Merck $\mathrm{KGaA}$ ) was added to cells to dissolve the formazan. The absorbance was detected at $490 \mathrm{~nm}$ with a microplate reader (Bio-Rad Laboratories, Inc.).

Reverse transcription-quantitative PCR (RT-qPCR). Total RNA from cells was extracted with mirVanaTM miRNA isolation kit (Thermo Fisher Scientific, Inc.), following the manufacturer's instructions. Subsequently, the target miRNA was reverse transcribed into cDNA using the TaqMan microRNA Reverse Transcription kit (Applied Biosystems; Thermo Fisher Scientific, Inc.), with the following parameters: $16^{\circ} \mathrm{C}$ for $30 \mathrm{~min}, 42^{\circ} \mathrm{C}$ for $30 \mathrm{~min}$ and $85^{\circ} \mathrm{C}$ for $5 \mathrm{~min}$. q-PCR was conducted using SYBR-Green (Applied Biosystems; Thermo Fisher Scientific, Inc.), following the amplification instructions. The following thermocycling conditions were used: $95^{\circ} \mathrm{C}$ for $5 \mathrm{~min} ; 40$ cycles at $95^{\circ} \mathrm{C}$ for $10 \mathrm{sec}, 60^{\circ} \mathrm{C}$ for $1 \mathrm{~min}$ and a final extension at $72^{\circ} \mathrm{C}$ for $5 \mathrm{~min}$. All primers were obtained from Sangon Biotech Co., Ltd.: miR-212-3p forward, 5'-GGTAACAGTCTCCAGTCA-3'; miR-212-3p reverse, 5'-GCAATTGCACTGGATACG-3'; U6 forward, 5'-CTC GCTTCGGCAGCACATATACT-3'; U6 reverse, 5'-CGCTT CACGAATTTGCGTGT-3'. Each sample was conducted in triplicate. The expression of miR-212-3p was normalized to U6 small nuclear RNA, using the $2^{-\Delta \Delta \mathrm{Cq}}$ method (16).

Cell apoptosis assay. Cell apoptosis was investigated by flow cytometry using the Annexin V-FITC/PI detection kit (Sigma-Aldrich; Merck KGaA). Following treatment with PTX for $24 \mathrm{~h}$, Huh7 and Huh7/PTX were resuspended in binding buffer and then incubated with $10 \mu \mathrm{l}$ Annexin V-FITC and PI for $20 \mathrm{~min}$ at room temperature in the dark. The positive cells (Annexin V-FITC ${ }^{+} / \mathrm{PI}^{-/+}$) were examined using a flow cytometer (BD Biosciences) and BD FACStation software (version 6.1; BD Biosciences)

Western blotting. Total protein was extracted using RIPA lysis buffer (Thermo Fisher Scientific, Inc.) containing $1 \%$ protease inhibitor and quantified by bicinchoninic acid protein assay kit (Sigma-Aldrich; Merck KGaA), according to the manufacturer's instructions. Denatured proteins (20 $\mu \mathrm{g} /$ lane) were loaded onto 6 or $10 \%$ SDS-PAGE gels and then transferred to polyvinylidene difluoride membranes (Millipore). Membranes were subsequently blocked in $5 \%$ non-fat milk for $1 \mathrm{~h}$ at room temperature. Membranes were then incubated with primary antibodies against p-glycoprotein (P-gp) (1:1,000; cat. no. ab235954), glutathione S-transferase $\pi$ (GST- $\pi)(1: 2,000$; cat. no. ab153949), E-cadherin (1:500; cat. no. ab15148), cytokeratin 18 (1:10,000; cat. no. ab133263), $\mathrm{N}$-cadherin $(1: 1,000$; cat. no. ab18203), vimentin $(1: 1,000$; cat. no. ab92547), ZEB2 (1:500, cat. no. ab138222) or $\beta$-actin (1:5,000; cat. no. ab227387) (all from Abcam) 
A

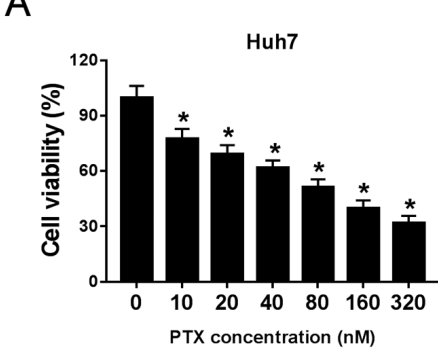

D

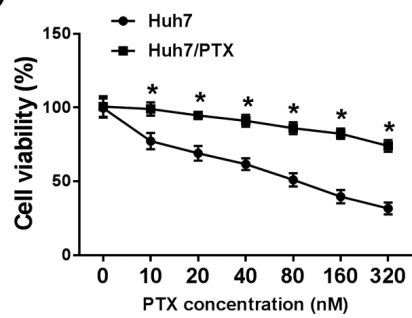

B

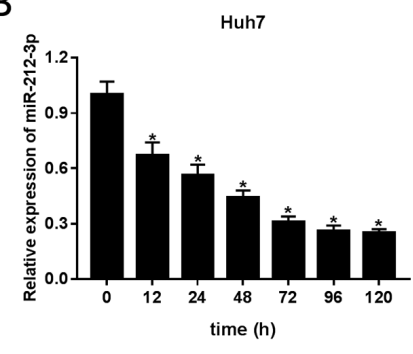

E

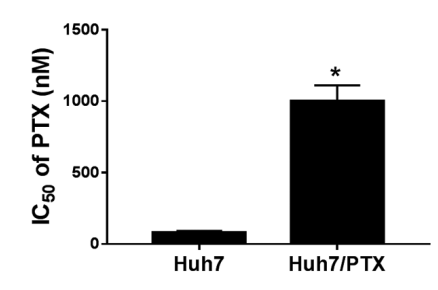

C

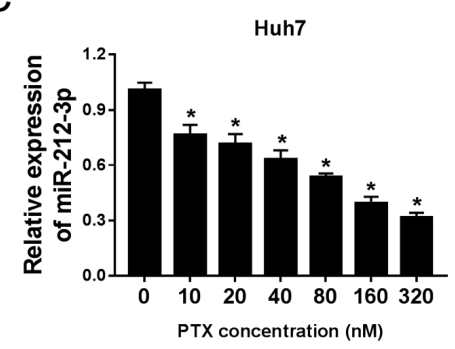

F

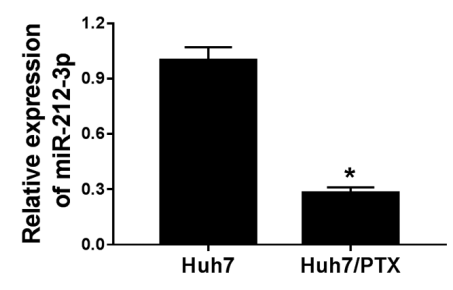

Figure 1. miR-212-3p expression was low in PTX-resistant hepatocellular carcinoma cells. (A) Cell viability was investigated in Huh7 cells treated with different concentrations of PTX for $24 \mathrm{~h}$ by MTT. (B and C) The expression of miR-212-3p was measured in PTX-treated Huh7 cells at different time points and different concentrations by reverse transcription-quantitative PCR. (D and E) The cell viability was detected in Huh7 and Huh7/PTX cells treated with varying concentrations of PTX for $24 \mathrm{~h}$, and the $\mathrm{IC}_{50}$ of PTX was analyzed. (F) The level of miR-212-3p was examined in Huh7 and Huh7/PTX cells. * $<0.05$ : A, B and C, PTX-treated group vs. non-treated group; D, E and F, Huh7/PTX group vs. Huh7 group. PTX, paclitaxel; miR, microRNA; IC ${ }_{50}$, half maximal inhibitory concentration.

overnight at $4^{\circ} \mathrm{C} . \beta$-actin was regarded as a housekeeping protein in this study. After three washes in Tris-buffer saline, containing $0.1 \%$ Tween-20 (TBST), membranes were incubated in horseradish peroxidase-conjugated secondary antibodies (1:10,000; cat. no. ab6721; Abcam) for $2 \mathrm{~h}$ at room temperature. The protein bands were visualized using enhanced chemiluminescence (ECL) chromogenic substrate (GE Healthcare) and analyzed using the Image Lab software (version 2.1; Bio-Rad Laboratories. Inc.).

Transwell assay. Cell migration and invasion were investigated by transwell assay. For cell invasion assay, chambers were pre-coated with Matrigel (BD Biosciences). Huh7 and Huh7/PTX cells $\left(2 \times 10^{4}\right.$ cells/well) were suspended in serum-free RPMI-1640 medium and then incubated in the upper chambers (Costar; Corning, Inc.) at $37^{\circ} \mathrm{C}$ with $5 \% \mathrm{CO}_{2}$ for $12 \mathrm{~h}$. Invasive cells on the basal side of the membrane were fixed with $4 \%$ paraformaldehyde for $20 \mathrm{~min}$ at room temperature, stained with $0.1 \%$ crystal violet (Sigma-Aldrich; Merck $\mathrm{KGaA}$ ) for $10 \mathrm{~min}$ at room temperature, and subsequently counted under a light microscope (magnification, x100) (Olympus Corporation). Migrated cells were investigated by using transwell chambers, following a similar approach, without Matrigel.

Luciferase assays. The putative binding sites of miR-212-3p and ZEB2 were predicted by TargetScan Human Release 7.1 (www.targetscan.org/vert_71/). The wild-type or mutant sequence of 3' untranslated regions (3'-UTR) of ZEB2 were amplified by PCR and cloned into the pGL3 luciferase reporter vector (Promega Corporation), in order to generate the ZEB2-Wt or ZEB2-Mut, respectively. Wt or Mut luciferase reporter vector was co-transfected with miR-212-3p mimic, miR-NC, miR-212-3p inhibitor or miR-NC inhibitor in Huh7 and Huh7/PTX cells, using Lipofectamine ${ }^{\mathrm{TM}} 2000$ (Invitrogen;
Thermo Fisher Scientific, Inc.), according to the manufacturer's protocols. Subsequently, cells were lysed and used for the luciferase activity analysis by using the Dual-Luciferase Assay kit (Promega Corporation) after $48 \mathrm{~h}$ of transfection. Firefly luciferase activity was normalized to Renilla luciferase activity.

Statistical analysis. Data are presented as the mean \pm standard deviation of three independent experiments. The differences were investigated by Student's t-test or one-way analysis of variance followed by Tukey's post hoc test using the GraphPad Prism 7 software (GraphPad Software, Inc.). P $<0.05$ was considered to indicate a statistically significant difference.

\section{Results}

miR-212-3p expression is downregulated in PTX-resistant HCC cells. Following treatment with different concentrations of PTX for $24 \mathrm{~h}$, the viability of Huh7 cells was significantly decreased in a concentration-dependent manner (Fig. 1A). Moreover, the data from the RTq-PCR assay revealed that the expression of miR-212-3p was progressively reduced in Huh7 cells after treatment with PTX at various time points or exposure to different doses at $24 \mathrm{~h}$ (Fig. 1B and C). In order to investigate the potential role of miR-212-3p in HCC resistance, PTX-resistant Huh7/PTX cells were constructed. MTT analysis showed that Huh7/PTX cells displayed higher cell viability compared with Huh7 cells in response to different concentrations of PTX (Fig. 1D). Meanwhile, the $\mathrm{IC}_{50}$ at $24 \mathrm{~h}$ of PTX was $\sim 80 \mathrm{nM}$ in Huh7 cells and 1,000 nM in Huh7/PTX cells (Fig. 1E). Furthermore, downregulation of miR-212-3p was demonstrated in Huh7/PTX cells compared with Huh7 cells (Fig. 1F). Furthermore, similar events were also observed in HCCLM3 cells (Fig. S1A-D). Taken together, dysregulated miR-212-3p may be associated with PTX resistance in HCC cells. 
A

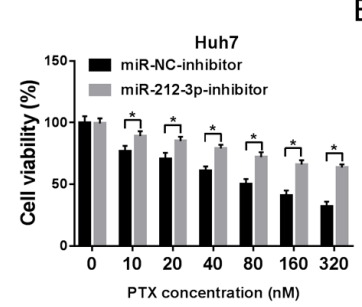

E

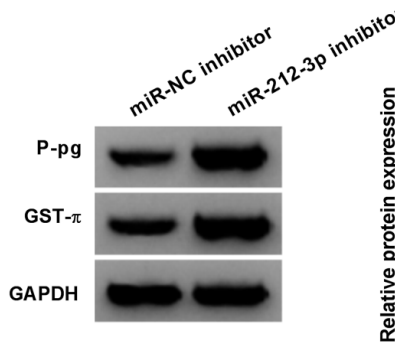

B

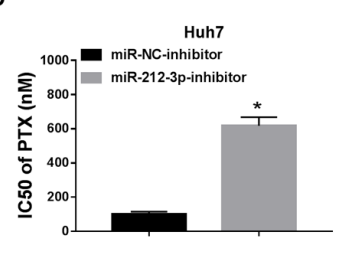

intor

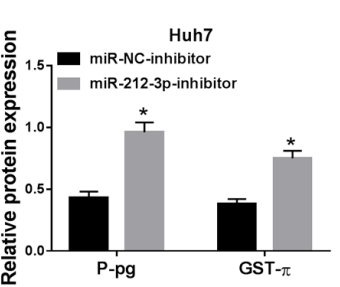

C

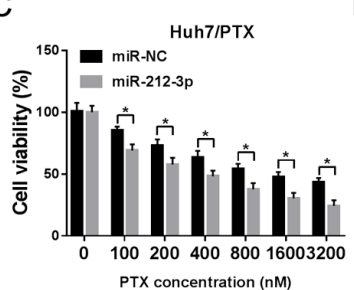

F

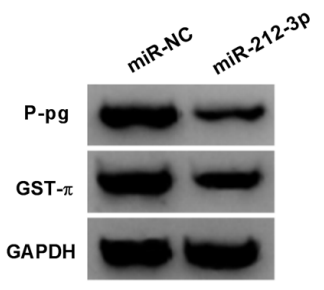

D
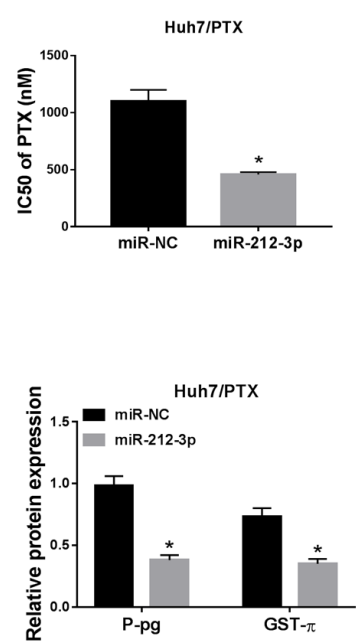

G

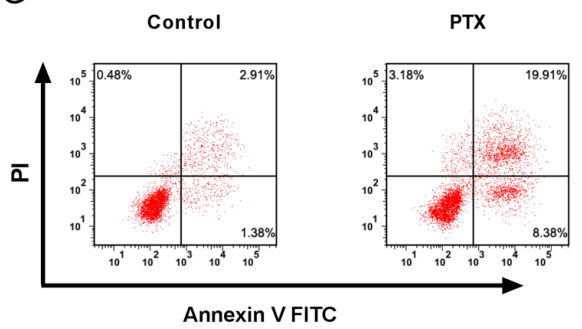

miR-NC-inhibitor+PTX
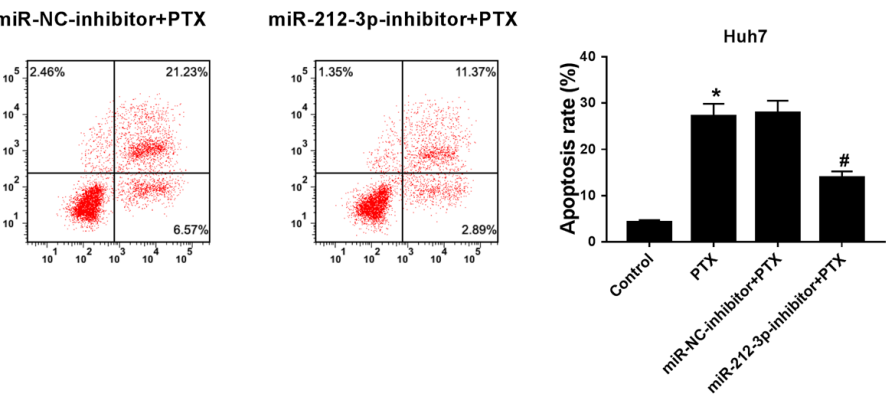

$\mathrm{H}$
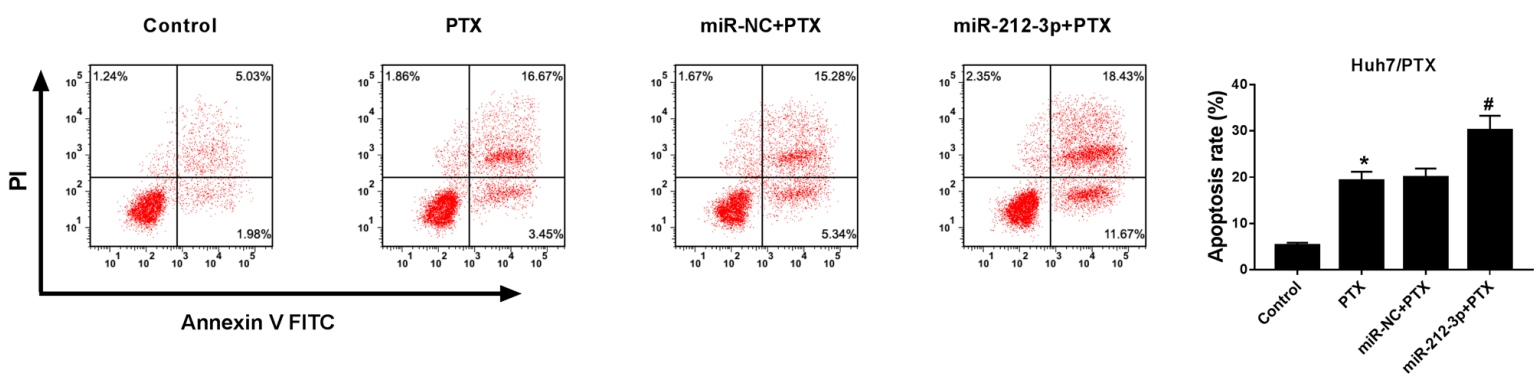

Figure 2. Addition of miR-212-3p inhibited PTX resistance in HCC cells. (A) The effect of miR-212-3p inhibition on cell viability was investigated in Huh7 cells treated with different concentrations of PTX for $24 \mathrm{~h}$. (B) The $\mathrm{IC}_{50}$ of PTX in Huh7 cells transfected with miR-212-3p inhibitor or miR-NC inhibitor. (C) The effect of miR-212-3p overexpression on cell viability was detected in Huh7/PTX cells treated with different concentrations of PTX for 24 h. (D) The $\mathrm{IC}_{50}$ of PTX in Huh7/PTX cells transfected with miR-212-3p or miR-NC. (E and F) The protein expression levels of P-gp and GST- $\pi$ were detected in the cells. (G) Cell apoptosis was evaluated in Huh7 cells treated with PTX and miR-212-3p inhibitor by flow cytometry. (H) The effect of miR-212-3p on PTX-induced apoptosis was analyzed in Huh7/PTX cells. ${ }^{*} \mathrm{P}<0.05 ;{ }^{~} \mathrm{P}<0.05$ : A, B and E, miR-212-3p inhibitor group vs. miR-NC inhibitor group; C, D and F, miR-212-3p group vs. miR-NC group; G and H, PTX group vs. control group, miR-212-3p inhibitor group + PTX vs. miR-NC inhibitor + PTX group, and miR-212-3p group + PTX vs. miR-NC + PTX group. PTX, paclitaxel; miR, microRNA; NC, negative control; IC $_{50}$, half maximal inhibitory concentration; $\mathrm{P}$-gp, p-glycoprotein; GST- $\pi$, glutathione S-transferase $\pi$.

miR-212-3p inhibits PTX resistance in HCC cells. To explore the function of miR-212-3p on PTX resistance in HCC cells, Huh7 cells were transfected with miR-NC inhibitor or miR-212-3p inhibitor and Huh7/PTX cells were transfected with miR-212-3p mimic or miR-NC, respectively. The transfection efficiency was confirmed (Fig. S2A and B). Following treatment with different concentrations of PTX, MTT assay demonstrated that the knockdown miR-212-3p reversed the inhibitory effect of PTX on the viability of Huh7 cells and led to increased $\mathrm{IC}_{50}$ of PTX from 85 to $624 \mathrm{nM}$ (Fig. 2A and B). However, the overexpression of miR-212-3p exacerbated PTX-mediated inhibition of cell viability in Huh7/PTX cells and resulted in reduced $\mathrm{IC}_{50}$ of PTX from
1,086 to $365 \mathrm{nM}$ (Fig. 2C and D). In addition, the protein levels of P-gp and GST- $\pi$ were significantly increased in Huh7 cells by miR-212-3p knockdown and decreased in Huh7/PTX cells following miR-212-3p overexpression (Fig. 2E and F). Moreover, cell apoptosis was measured in Huh7 and Huh7/PTX cells, following treatment with 80 or 1,000 nM PTX for $24 \mathrm{~h}$. The results demonstrated that treatment with $80 \mathrm{nM}$ PTX induced Huh7 cells apoptosis, whereas miR-212-3p depletion protected cells from PTX-induced apoptosis (Fig. 2G). Furthermore, the introduction of miR-212-3p aggravated PTX-induced cells apoptosis in Huh7/PTX cells (Fig. 2H). Overall, these findings suggest that miR-212-3p attenuates PTX resistance in HCC cells. 


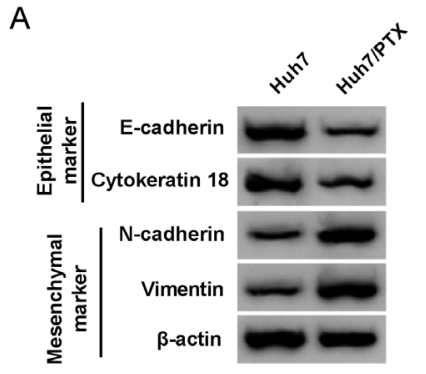

C

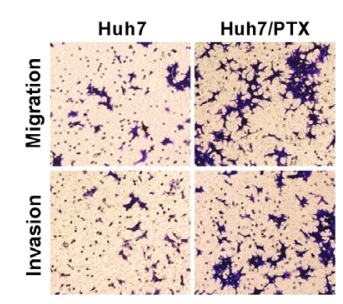

B
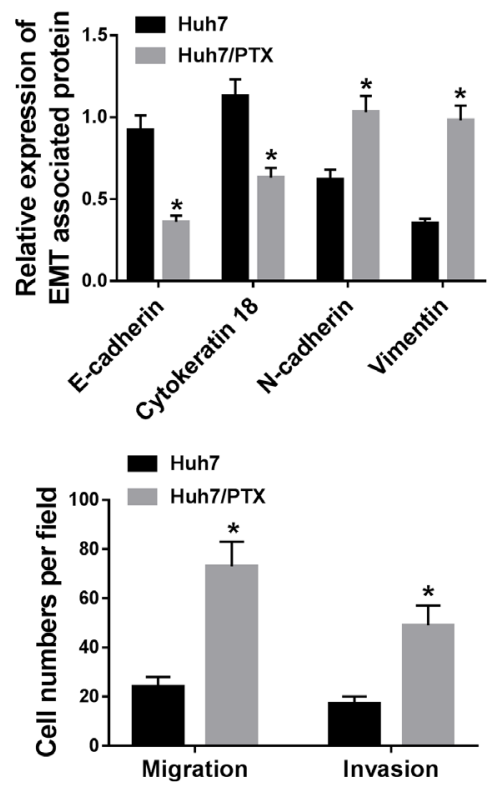

Figure 3. EMT, migration and invasion are enhanced in PTX-resistant hepatocellular carcinoma cells. (A and B) The protein expression levels of E-cadherin, cytokeratin 18, N-cadherin and vimentin were measured in Huh7 and Huh7/PTX cells by western blotting. (C and D) Cell migration and invasion were examined in Huh7 and Huh7/PTX cells by transwell assay. "P<0.05, Huh7/PTX group vs. with Huh7 group. EMT, epithelial-mesenchymal transition; PTX, paclitaxel.

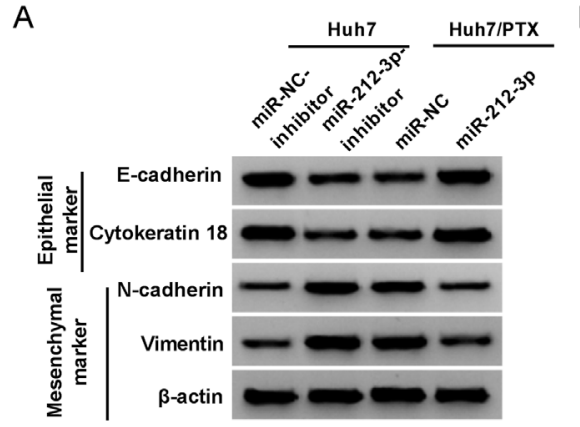

C

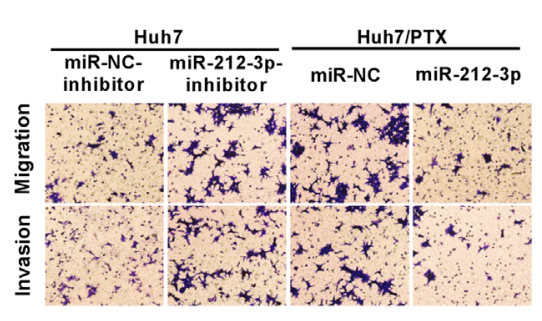

B

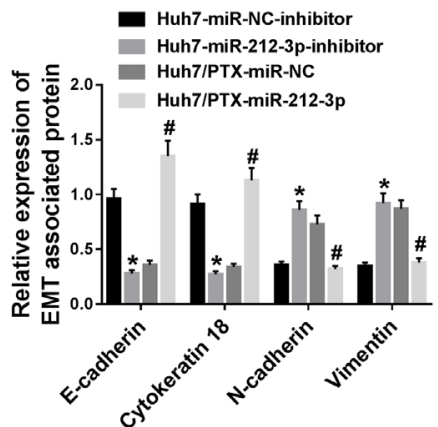

D

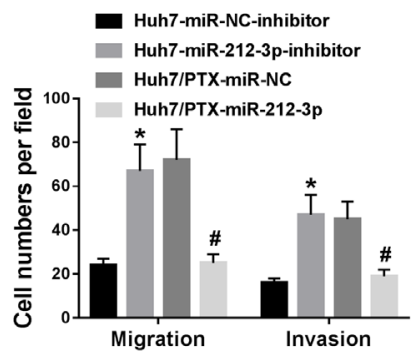

Figure 4. miR-212-3p overexpression suppresses EMT, migration and invasion in PTX-resistant hepatocellular cells. (A and B) The effect of miR-212-3p on E-cadherin, cytokeratin 18, N-cadherin and vimentin protein expression levels was investigated in Huh7 and Huh7/PTX cells, following transfection with miR-212-3p inhibitor or miR-212-3p. (C and D) Cell migration and invasion were measured in miR-212-3p inhibitor-transfected Huh7 and miR-212-3p-transfected Huh7/PTX cells. " $\mathrm{P}<0.05$, miR-212-3p inhibitor group vs. miR-NC inhibitor group; ${ }^{~} \mathrm{P}<0.05$, miR-212-3p group vs. miR-NC group. PTX, paclitaxel; miR, microRNA; NC, negative control; EMT, epithelial-mesenchymal transition.

EMT, migration and invasion are associated with PTX resistance in HCC cells. Since EMT, migration and invasion may play essential roles in chemoresistance, these processes were assessed in Huh7 and Huh7/PTX cells. The protein levels of EMT markers were investigated by western blotting. The results demonstrated decreased E-cadherin and cytokeratin 18, whilst increased N-cadherin and vimentin, in Huh7/PTX cells compared with Huh7 cells, suggesting that PTX resistance promoted EMT (Fig. 3A and B). Furthermore, cell migration and invasion were detected in HCC cells. The transwell assays demonstrated increased cell migration and invasion abilities in Huh7/PTX cells compared with PTX-sensitive 
A

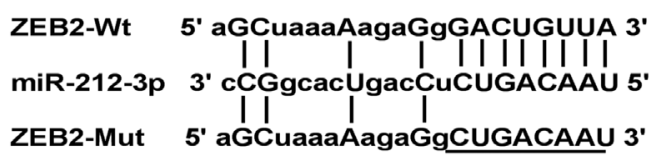

B

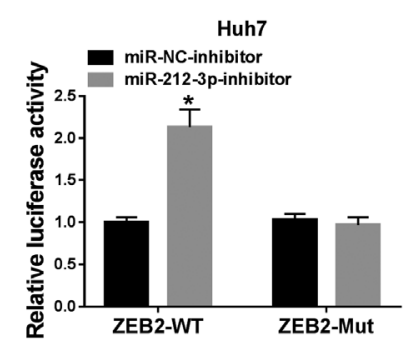

D

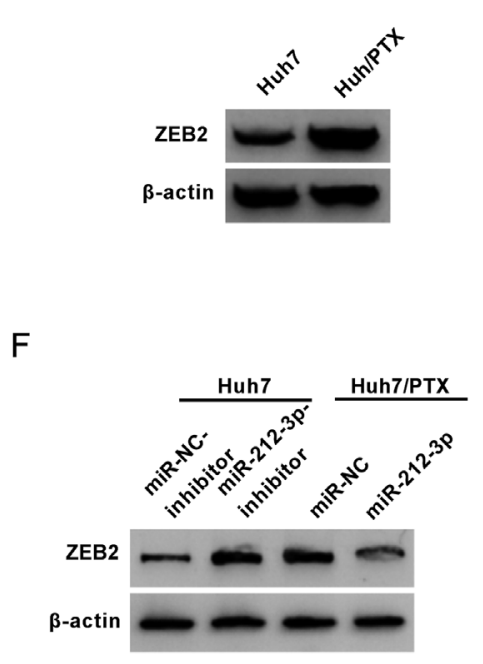

C

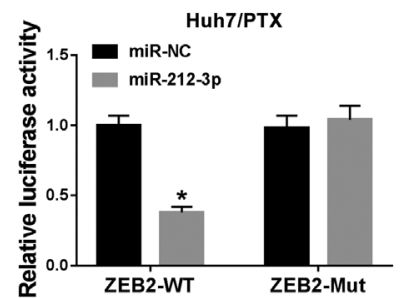

$\mathrm{E}$

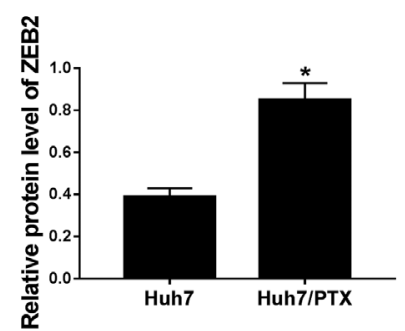

G

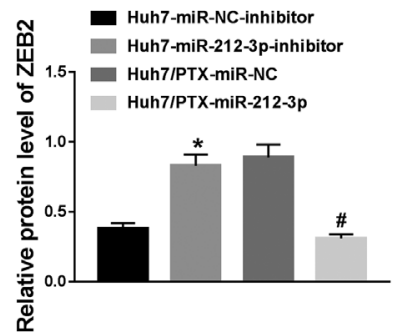

Figure 5. ZEB2 is a direct target of miR-212-3p. (A) The potential binding sites of miR-212-3p and ZEB2 were predicted by TargetScan Human Release 7.1. (B) The luciferase activity was investigated in Huh7 cells co-transfected with miR-212-3p inhibitor or miR-NC inhibitor and ZEB2-WT or ZEB2-Mut. (C) The luciferase activity was evaluated in Huh7/PTX cells co-transfected with miR-212-3p or miR-NC and ZEB2-WT or ZEB2-Mut. (D and E) The protein expression level of ZEB2 was detected in Huh7 and Huh7/PTX cells by western blotting. (F and G) The effect of miR-212-3p on ZEB2 expression level was investigated in miR-212-3p inhibitor-transfected Huh7 and miR-212-3p-transfected Huh7/PTX cells. "P<0.05: B and G, miR-212-3p inhibitor group vs. miR-NC inhibitor group; C, miR-212-3p group vs. miR-NC group; E, Huh7/PTX group vs. Huh7 group. " $\mathrm{P}<0.05$ : G, miR-212-3p group vs. miR-NC group. PTX, paclitaxel; miR, microRNA; NC, negative control; ZEB, zinc finger E-box binding homeobox 2; WT, wild type; Mut, mutant.

cells (Fig. 3C and D). Overall, these results suggest that EMT, migration and invasion are positively associated with PTX resistance in HCC cells.

miR-212-3p controls PTX resistance by regulating EMT, migration and invasion in HCC cells. Based on the aforementioned results, the effect of miR-212-3p on EMT, migration and invasion was investigated in Huh7 and Huh7/PTX cells. Western blotting assay showed that miR-212-3p deficiency promoted EMT in Huh7 cells, demonstrated by decreased E-cadherin, cytokeratin 18 and increased of $\mathrm{N}$-cadherin, vimentin expression levels (Fig. 4A and B). However, the overexpression of miR-212-3p resulted in the opposite effects on EMT in Huh7/PTX cells (Fig. 4A and B). Moreover, inhibition of miR-212-3p resulted in increased migration and invasion abilities of Huh7 cells, whereas its addition blocked cell migration and invasion in (Fig. 4C and D). These results indicate that
miR-212-3p regulates PTX resistance by suppressing EMT, migration and invasion in HCC cells.

ZEB2 is a target of miR-212-3p. Given that functional miRNAs exert their effects by regulating target genes, the targets of miR-212-3p were predicted by TargetScan Human Release 7.1 online. Potential binding sites of miR-212-3p were predicted in ZEB2, suggesting that ZEB2 might be a target of miR-212-3p (Fig. 5A). Hence, the prediction was next validated by luciferase activity assay. Results showed that the knockdown of miR-212-3p resulted in enhanced luciferase activity in ZEB2-Wt-transfected Huh7 cells, while little effect was observed with ZEB2-Mut cells (Fig. 5B). Moreover, a notable decrease in luciferase activity was demonstrated in Huh7/PTX cells co-transfected with miR-212-3p and ZEB2-Wt, whereas the effect was lost in the ZEB2-Mut group (Fig. 5C). Besides, ZEB2 protein expression was significantly enhanced in 
A

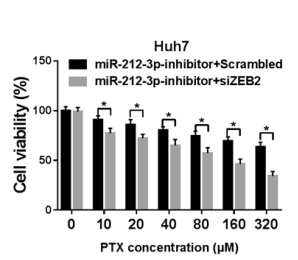

E

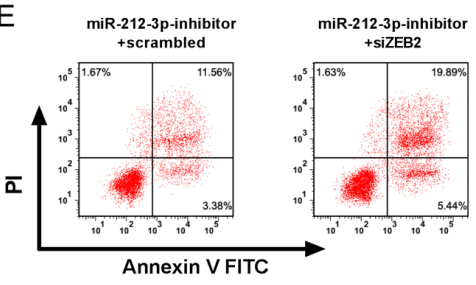

F

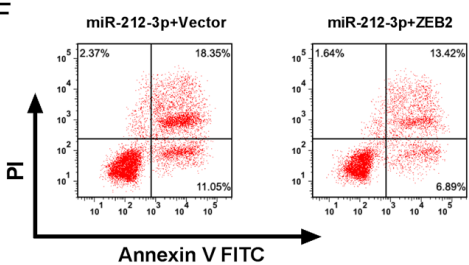

Annexin V FITC
B
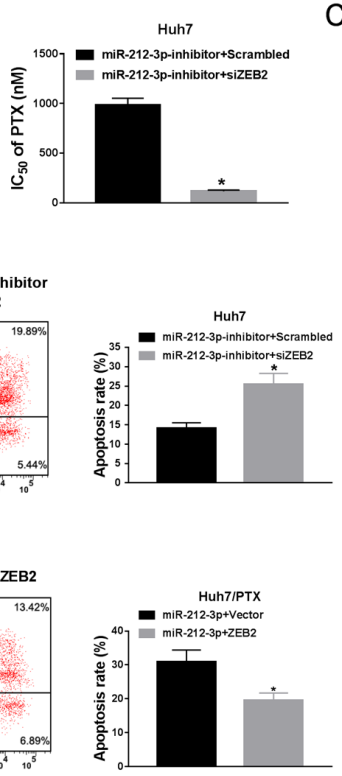

C

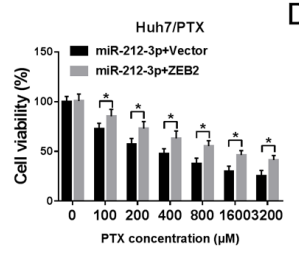

G

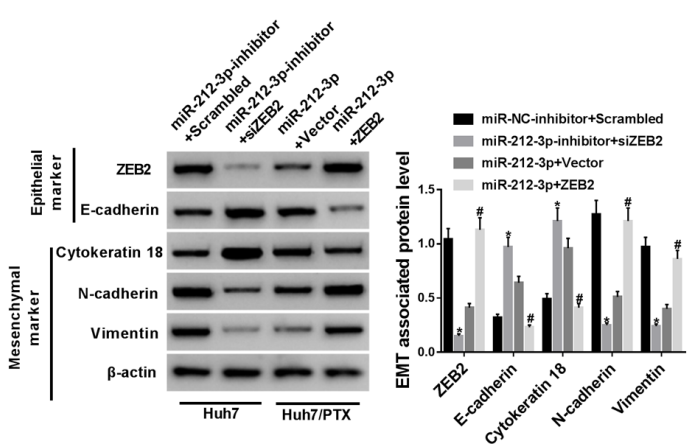

$\mathrm{H}$

I
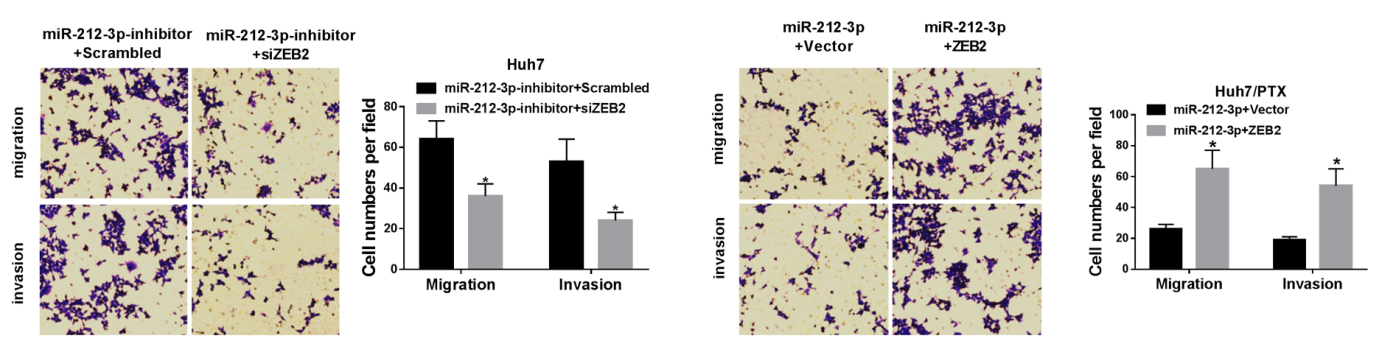

Figure 6. ZEB2 is required for miR-212-3p-mediated PTX resistance. Huh7 cells were co-transfected with miR-212-3p inhibitor and scrambled or siZEB2. Huh7/PTX cells were co-transfected with miR-212-3p and vector or ZEB2. (A-D) Cell viability and $\mathrm{IC}_{50}$ of PTX were measured in transfected Huh7 and Huh7/PTX cells after treatment with different concentrations of PTX for $24 \mathrm{~h}$. (E and F) Cell apoptosis was detected in transfected Huh7 and Huh7/PTX cells after treatment with the corresponding concentration ( $80 \mathrm{nM}$ for Huh7 cells and 1,000 nM for Huh7/PTX cells) of PTX for $24 \mathrm{~h}$. (G) The protein levels of ZEB2, E-cadherin, cytokeratin 18, N-cadherin and vimentin were examined in transfected Huh7 and Huh7/PTX cells. (H and I) Cell migration and invasion were analyzed in transfected Huh7 and Huh7/PTX cells, respectively. ${ }^{*} \mathrm{P}<0.05$ : A, B, E, G and H, miR-212-3p inhibitor + siZEB2 group vs. miR-212-3p inhibitor + scrambled group; C, D, F and I, miR-212-3p + ZEB2 group vs. miR-212-3p + vector group. "P<0.05: G, miR-212-3p + ZEB2 group vs. miR-212-3p + vector group. PTX, paclitaxel; miR, microRNA; ZEB, zinc finger E-box binding homeobox 2; $\mathrm{IC}_{50}$, half maximal inhibitory concentration.

Huh7/PTX compared with Huh7 cells (Fig. 5D and E). In addition, the effect of miR-212-3p on ZEB2 expression was also investigated in Huh7 and Huh7/PTX cells. As shown in Fig. 5F and G, miR-212-3p abrogation induced ZEB2 expression in Huh7 cells, while miR-212-3p overexpression blocked ZEB2 abundance in Huh7/PTX cells. These findings suggest ZEB2 as a direct target of miR-212-3p in HCC cells.

ZEB2 is involved in miR-212-3p-mediated PTX resistance in HCC cells. In order to assess whether ZEB2 was required for miR-212-3p-mediated PTX resistance, Huh7 cells were co-transfected with miR-212-3p inhibitor and siZEB2 or scrambled siRNA and Huh7/PTX cells were co-transfected with miR-212-3p and ZEB2 or vector. The transfection efficacy was confirmed (Fig. S3A-D). Cell viability and apoptosis were detected in transfected cells, following treatment with PTX for $24 \mathrm{~h}$. MTT assay showed that the silencing of ZEB2 decreased cell viability and $\mathrm{IC}_{50}$ of PTX in Huh7 cells, whereas ZEB2 restoration protected the cell viability and increased $\mathrm{IC}_{50}$ of PTX in Huh7/PTX cells (Fig. 6A-D). Moreover, data from flow cytometry reflected that the absence of ZEB2 induced cell apoptosis in miR-212-3p-deficient Huh7 cells, while the introduction of ZEB2 weakened miR-212-3p-induced apoptosis in Huh7/PTX cells (Fig. 6E and F). In addition, a significant loss of ZEB2 protein expression was demonstrated in siZEB2-transfected Huh7 cells and a notable increase in ZEB2 expression was displayed in Huh7/PTX cells transfected with ZEB2 overexpression vector, compared with their corresponding control, respectively (Fig. 6G). Furthermore, ZEB2 interference overturned the effect of miR-212-3p inhibition on EMT, revealed by elevated E-cadherin, cytokeratin 18 and reduced $\mathrm{N}$-cadherin and vimentin (Fig. 6G) expression levels. However, ZEB2 overexpression indicated opposite trends in miR-212-3p-transfected Huh7/PTX cells (Fig. 6G). Besides, ZEB2 abrogation inhibited cell migration and invasion in Huh7 cells transfected with miR-212-3p inhibitor and siZEB2 compared with cells transfected with miR-212-3p inhibitor and scrambled (Fig. 6H). Conversely, ZEB2 restoration promoted cell migration and invasion in miR-212-3p-transfected Huh7/PTX cells (Fig. 6I). Collectively, these results indicate that miR-212-3p mediates PTX resistance by targeting ZEB2 in HCC cells. 


\section{Discussion}

The efficacy of chemotherapy is unsatisfactory, owing to chemoresistance in HCC (3). PTX, as an important chemotherapeutic agent, has been widely used for the treatment of HCC (17). In the present study, Huh7 cells were treated with PTX in order to establish Huh7/PTX cells. MTT analysis showed that PTX treatment suppressed cell viability of Huh7 and Huh7/PTX cells, and Huh7/PTX cells had higher viability and $\mathrm{IC}_{50}$ of PTX, suggesting the anti-HCC role of PTX and successful establishment of PTX-resistant HCC cells. The present study was the first to demonstrate that miR-212-3p inhibited PTX resistance by targeting ZEB2 in HCC cells.

A previous study suggested that miR-212-3p was downregulated in HCC (12), while the effect of miR-212-3p on PTX resistance was unclear. In the present study, miR-212-3p expression was decreased in Huh7 cells, following treatment with PTX, and Huh7/PTX cells displayed lower expression level of miR-212-3p, suggesting that miR-212-3p may play an important role in PTX resistance in HCC cells. Moreover, following treatment with PTX, analyses by MTT, western blotting and flow cytometry revealed that miR-212-3p decreased PTX resistance in HCC cells, indicating miR-212-3p as a promising target for improving the efficacy of chemotherapy in HCC.

Several mechanisms, such as EMT, inflammation, autophagy and oxidative stress, play key roles in regulating drug resistance via varying pathways (18). EMT has been reported to be involved in the advancement of HCC and is associated with the development of drug resistance in advanced HCC (19). The expression levels of EMT markers showed that EMT was induced in Huh7/PTX cells, suggesting that EMT was positively associated with PTX resistance. This is consistent with a previous study that also indicated the association between EMT and PTX resistance by regulating the expression levels of E-cadherin, cytokeratin 18, N-cadherin and vimentin (20). Moreover, it was found that miR-212-3p inhibited PTX resistance by regulating EMT. Furthermore, current evidence indicates that EMT is associated with cell migration and invasion in HCC (21). Furthermore, the acquisition of PTX resistance was suggested to be associated with metastatic properties $(22,23)$. In the present study, the contribution of migration and invasion to PTX resistance was demonstrated, and the potential role of miR-212-3p in suppressing drug resistance by regulating migration and invasion.

miRNAs function by mediating the expression of target genes in different conditions. For example, miR-212-3p was reported to inhibit cell proliferation and induce apoptosis by regulating FOXA1 expression in osteosarcoma (9). Moreover, miR-212-3p mediated cell proliferation, migration, cell cycle and EMT by targeting Ras-associated binding-GTPase 1a (Rabla) in intrahepatic cholangiocarcinoma, in response to hypoxia treatment (24). Besides, miR-212-3p may inhibit proliferation and increase apoptosis via suppressing sex determining region Y-box 5 (SOX5) in rheumatoid arthritis (25). Apart from these, serum and glucocorticoid-inducible kinase 3 (SGK3) was also a target of miR-212-3p, which regulated cell proliferation in glioblastoma (26). ZEB2, an
EMT-associated gene, induced EMT to regulate cell migration and invasion in HCC by targeting miR-145 (15). In addition, miR-139-5p also inhibited EMT, migration and invasion by mediating ZEB2 in HCC (27). Promisingly, it was found that miR-212-3p could also target ZEB2 in HCC cells, which was identified by luciferase activity analysis. Western blotting showed increased protein expression level of ZEB2 in PTX-resistant HCC cells compared with PTX-sensitive cells, suggesting that ZEB2 may contribute towards PTX resistance in HCC cells, which is similar to findings of other studies, which demonstrated positive association between ZEB2 and EMT, and supported chemoresistance in lung cancer $(14,28)$. Furthermore, rescue experiments demonstrated that ZEB2 interference or restoration reversed miR-212-3p knockdown or overexpression-mediated regulation of PTX resistance. These data reflected that miR-212-3p counteracted PTX resistance by targeting ZEB2 in HCC cells. In the present study, the potential mechanism underlying the regulation of PTX resistance by miR-212-3p was only investigated in vitro. Hence, a preclinical study with an animal model, as well as clinical experiments are expected in future.

In conclusion, low expression of miR-212-3p was demonstrated in PTX-resistant HCC cells, and overexpression of miR-212-3p inhibited PTX resistance through regulating EMT, migration and invasion by targeting ZEB2 in HCC cells. Thus, the present study provides a novel regulator for studying the efficacy of chemotherapy in HCC.

\section{Acknowledgements}

Not applicable.

\section{Funding}

No funding was received.

\section{Availability of data and materials}

All data generated or analyzed during the present study are included in this published article.

\section{Authors' contributions}

JY conceived the present study. RC and YL contributed to manuscript preparation and data analyses, with constructive discussions. All authors performed the experiments. JY performed the data analyses and drafted the initial manuscript. All authors read and approved the final manuscript.

\section{Ethics approval and consent to participate}

Not applicable.

\section{Patient consent for publication}

Not applicable.

\section{Competing interests}

The authors declare that they have no competing interests. 


\section{References}

1. Craig AJ, von Felden J, Garcia-Lezana T, Sarcognato S and Villanueva A: Tumour evolution in hepatocellular carcinoma. Nat Rev Gastroenterol Hepatol 17: 139-152, 2020.

2. Forner A, Reig M and Bruix J: Hepatocellular carcinoma. Lancet 391: 1301-1314, 2018.

3. Lohitesh K, Chowdhury R and Mukherjee S: Resistance a major hindrance to chemotherapy in hepatocellular carcinoma: An insight. Cancer Cell Int 18: 44, 2018.

4. Meena AS, Sharma A, Kumari R, Mohammad N, Singh SV and Bhat MK: Inherent and acquired resistance to paclitaxel in hepatocellular carcinoma: Molecular events involved. PLoS One 8: e61524, 2013.

5. Vasuri F, Visani M, Acquaviva G, Brand T, Fiorentino M, Pession A, Tallini G, D'Errico A and de Biase D: Role of microRNAs in the main molecular pathways of hepatocellular carcinoma. World J Gastroenterol 24: 2647-2660, 2018.

6. Yan H, Wang S, Yu H, Zhu J and Chen C: Molecular pathways and functional analysis of miRNA expression associated with paclitaxel-induced apoptosis in hepatocellular carcinoma cells. Pharmacology 92: 167-174, 2013.

7. Huang X, Qin J and Lu S: Up-regulation of miR-877 induced by paclitaxel inhibits hepatocellular carcinoma cell proliferation though targeting FOXM1. Int J Clin Exp Pathol 8: 1515-1524, 2015 .

8. Chen Y, Feng F, Gao X, Wang C, Sun H, Zhang C, Zeng Z, Lu Y, An L, Qu J, et al: MiRNA153 Reduces Effects of Chemotherapeutic Agents or Small Molecular Kinase Inhibitor in HCC Cells. Curr Cancer Drug Targets 15: 176-187, 2015.

9. Xie C, Chen B, Wu B, Guo J and Cao Y: LncRNA TUG1 promotes cell proliferation and suppresses apoptosis in osteosarcoma by regulating miR-212-3p/FOXA1 axis. Biomed Pharmacother 97: $1645-1653,2018$.

10. He X and Fan S: hsa-miR-212 modulates the radiosensitivity of glioma cells by targeting BRCA1. Oncol Rep 39: 977-984, 2018.

11. Tu H, Wei G, Cai Q, Chen X, Sun Z, Cheng C, Zhang L, Feng Y, Zhou H, Zhou B, et al: MicroRNA-212 inhibits hepatocellular carcinoma cell proliferation and induces apoptosis by targeting FOXA1. Onco Targets Ther 8: 2227-2235, 2015.

12. Shen S, Lin Y, Yuan X, Shen L, Chen J, Chen L, Qin L and Shen B: Biomarker MicroRNAs for Diagnosis, Prognosis and Treatment of Hepatocellular Carcinoma: A Functional Survey and Comparison. Sci Rep 6: 38311, 2016.

13. Hegarty SV, Sullivan AM and O'Keeffe GW: Zeb2: A multifunctional regulator of nervous system development. Prog Neurobiol 132: 81-95, 2015.

14. Han ML, Zhao YF, Tan CH, Xiong YJ, Wang WJ, Wu F, Fei Y Wang L and Liang ZQ: Cathepsin L upregulation-induced EMT phenotype is associated with the acquisition of cisplatin or paclitaxel resistance in A549 cells. Acta Pharmacol Sin 37: 1606-1622, 2016

15. Li C, Lu L, Feng B, Zhang K, Han S, Hou D, Chen L, Chu X and Wang R: The lincRNA-ROR/miR-145 axis promotes invasion and metastasis in hepatocellular carcinoma via induction of epithelial-mesenchymal transition by targeting ZEB2. Sci Rep 7: $4637,2017$.

16. Livak KJ and Schmittgen TD: Analysis of relative gene expression data using real-time quantitative PCR and the 2(-Delta Delta C(T)) method. Methods 25: 402-408, 2001.
17. Zhu D, Wu S, Hu C, Chen Z, Wang H, Fan F, Qin Y, Wang C, Sun H, Leng X, et al: Folate-targeted polymersomes loaded with both paclitaxel and doxorubicin for the combination chemotherapy of hepatocellular carcinoma. Acta Biomater 58: 399-412, 2017.

18. Chen J, Jin R, Zhao J, Liu J, Ying H, Yan H, Zhou S, Liang Y, Huang D, Liang X, et al: Potential molecular, cellular and microenvironmental mechanism of sorafenib resistance in hepatocellular carcinoma. Cancer Lett 367: 1-11, 2015.

19. Mir N, Jayachandran A, Dhungel B, Shrestha R and Steel JC: Epithelial-to-Mesenchymal Transition: A Mediator of Sorafenib Resistance in Advanced Hepatocellular Carcinoma. Curr Cancer Drug Targets 17: 698-706, 2017.

20. Zhao YF, Han ML, Xiong YJ, Wang L, Fei Y, Shen X, Zhu Y and Liang ZQ: A miRNA-200c/cathepsin L feedback loop determines paclitaxel resistance in human lung cancer A549 cells in vitro through regulating epithelial-mesenchymal transition. Acta Pharmacol Sin 39: 1034-1047, 2018.

21. Zhang Y, Wang W, Wang Y, Huang X, Zhang Z, Chen B, Xie W, Li S, Shen S and Peng B: NEK2 promotes hepatocellular carcinoma migration and invasion through modulation of the epithelial-mesenchymal transition. Oncol Rep 39: 1023-1033, 2018.

22. Kim JJ, Yin B, Christudass CS, Terada N, Rajagopalan K, Fabry B, Lee DY, Shiraishi T, Getzenberg RH, Veltri RW, et al: Acquisition of paclitaxel resistance is associated with a more aggressive and invasive phenotype in prostate cancer. J Cell Biochem 114: 1286-1293, 2013.

23. Li H, Zhang P, Sun X, Sun Y, Shi C, Liu H and Liu X: MicroRNA-181a regulates epithelial-mesenchymal transition by targeting PTEN in drug-resistant lung adenocarcinoma cells. Int J Oncol 47: 1379-1392, 2015.

24. Hou P, Kang Y and Luo J: Hypoxia-mediated miR-212-3p downregulation enhances progression of intrahepatic cholangiocarcinoma through upregulation of Rabla. Cancer Biol Ther 19: 984-993, 2018

25. Liu Y, Zhang XL, Li XF, Tang YC and Zhao X: miR-212-3p reduced proliferation, and promoted apoptosis of fibroblast-like synoviocytes via down-regulating SOX5 in rheumatoid arthritis. Eur Rev Med Pharmacol Sci 22: 461-471, 2018.

26. Liu H, Li C, Shen C, Yin F, Wang K, Liu Y, Zheng B, Zhang W, Hou X, Chen X, et al: MiR-212-3p inhibits glioblastoma cell proliferation by targeting SGK3. J Neurooncol 122: 431-439, 2015.

27. Qiu G, Lin Y, Zhang H and Wu D: miR-139-5p inhibits epithelial-mesenchymal transition, migration and invasion of hepatocellular carcinoma cells by targeting ZEB1 and ZEB2. Biochem Biophys Res Commun 463: 315-321, 2015.

28. Duan X, Fu Z, Gao L, Zhou J, Deng X, Luo X, Fang W and Luo R: Direct interaction between miR-203 and ZEB2 suppresses epithelial-mesenchymal transition signaling and reduces lung adenocarcinoma chemoresistance. Acta Biochim Biophys Sin (Shanghai) 48: 1042-1049, 2016.

This work is licensed under a Creative Commons Attribution-NonCommercial-NoDerivatives 4.0 International (CC BY-NC-ND 4.0) License. 